\title{
Descrição de uma ferramenta digital e de um ambiente virtual para fins de segunda opinião em oftalmologia
}

\author{
Description of a digital image system and a virtual environment to be used as a remote \\ second referraloption in ophthalmology
}

\author{
Alessandro Dantas Pennella ${ }^{1}$ \\ Paulo Schor ${ }^{2}$ \\ Roberto Roizenblatt ${ }^{3}$
}

Trabalho realizado na Universidade Federal de São Paulo - UNIFESP.

${ }^{1}$ Estagiário do setor de Bioengenharia Ocular, Pósgraduando do Departamento de Oftalmologia da Universidade Federal de São Paulo - UNIFESP.

${ }^{2}$ Professor Afiliado da Universidade Federal de São Paulo - UNIFESP.

${ }^{3}$ Estagiário do Setor de Bioengenharia Ocular, Pósgraduando do Departamento de Oftalmologia da Universidade Federal de São Paulo - UNIFESP.

Endereço para correspondência: Rua Botucatu, 824 São Paulo (SP) CEP 04023-062

E-mail: sandrodp@hotmail.com

Recebido para publicação em 06.06.2002 Aceito para publicação em 26.04.2003

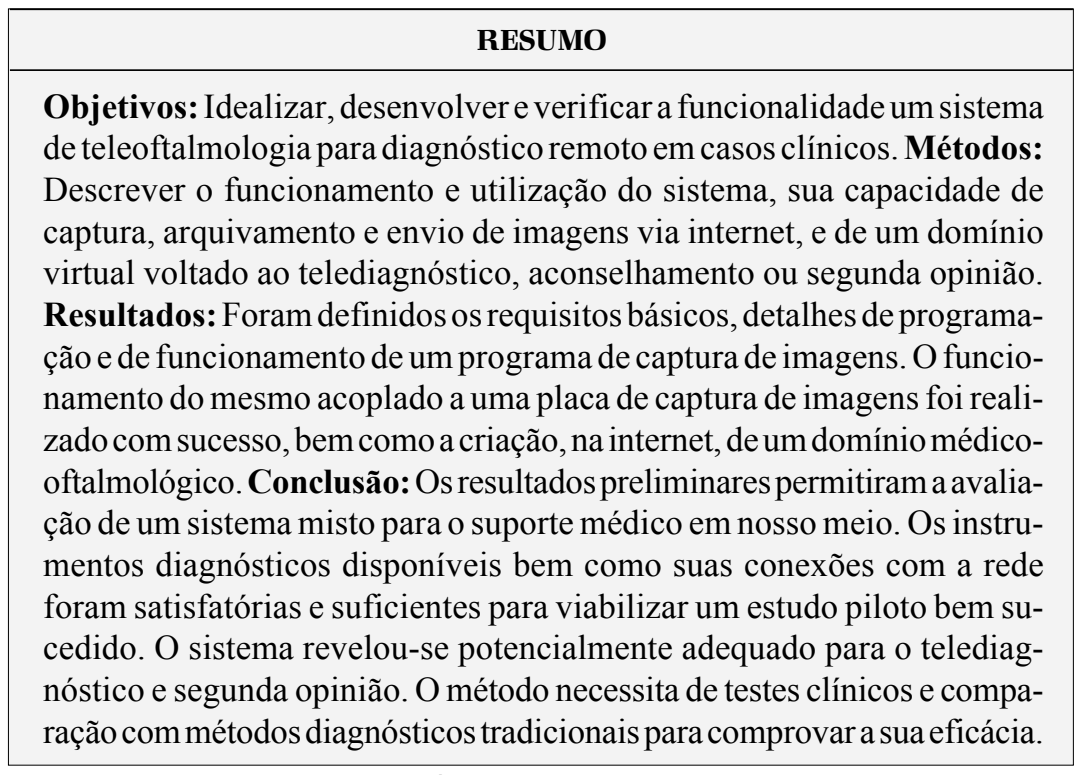

Descritores: Telemedicina; Diagnóstico por computador; Redes de comunicação de computadores

\section{INTRODUÇ̃̃O}

O aprimoramento das tecnologias de telecomunicação e informática nos anos 80 e da internet nos anos $90^{(1-2)}$ propiciou um ambiente favorável para a troca de informações. A utilização da internet torna-se praticamente compulsória para estes fins dada a facilidade e o baixo custo da transmissão das informações, permitindo uma eficiente veiculação e troca de imagens, sons e textos a partir de praticamente qualquer computador pessoal ${ }^{(3)}$. Os serviços de aconselhamento diagnóstico já existem em áreas como a cardiologia, radiologia e dermatologia ${ }^{(4-6)}$. A oftalmologia, por ser uma especialidade cujo diagnóstico se baseia preferencialmente em imagens, tem uma vocação natural para esta modalidade ${ }^{(7)}$. A tecnologia para ampla adesão da classe oftalmológica à telemedicina está disponível ${ }^{(8)}$.

Existem sistemas de captura de imagens comercialmente disponíveis no Brasil como o IMAGEnet ${ }^{\circledR}$ (Topcon-EUA) e o sistema Eye $Q^{\circledR}$ (CanonJapão), ambos com capacidade de capturar, armazenar e editar imagens. A diferença básica entre a interface para a internet do sistema $E y e Q^{\circledR}$, e o Eyemail $^{\circledR}$, proposto neste artigo, deve-se ao fato de que o sistema Eye $Q^{\circledR}$ envia as informações através do correio eletrônico e o Eyemail ${ }^{\circledR}$ via página HTML. A empresa alemã Siemens (www.siemens.com) descreve um sistema 
de teleradiologia que se baseia no envio de imagens digitalizadas com a descrição do caso clínico, via e-mail, para o domínio virtual de referência e as consultorias são acompanhadas de um arquivo digital de voz anexado às imagens. O mesmo sistema é utilizado no Brasil pela Rede $\mathrm{MD}^{\circledR}$ (www.redemd.com.br), sendo que neste caso as consultas podem retornar em até 3 dias. Existem vários sistemas de captura de imagem e banco de dados desenvolvidos no Brasil como o MedPlus ${ }^{\circledR}$, que apesar de ter a capacidade de capturar imagens não tem a interface para telemedicina desenvolvida para o Eyemail. Esta interface se expressa na capacidade de criar automaticamente páginas HTML, enviando diretamente as informações para o domínio virtual denominado suporte médico (www.suportemedico.com.br). Este website trabalha de forma síncrona (em tempo real) e permite o fornecimento imediato de respostas aos questionamentos dos usuários e discussão do caso clínico através de um sistema de interação virtual escrito (chat). O software Eyemail também pode enviar as informações diretamente para um endereço de correio eletrônico previamente configurado.

Os sistemas de videoconferência têm sido amplamente testados como ferramenta para a telemedicina. Trabalho realizado entre o Satakunta Central Hospital (Pori - Finlândia) e o Noormarkkku Health Center (Noormarkkku - Finlândia), que têm uma distância entre si de $15 \mathrm{Km}$, teve como objetivo avaliar as possibilidades técnicas para a realização de diagnóstico cirúrgico, via telemedicina, em 50 pacientes do sexo feminino, utilizando o sistema de teleconferência ${ }^{(9)}$. O método descrito valeu-se de um clínico geral, situado em um serviço distante dos grandes centros e sem um médico cirurgião, e de um sistema de teleconferência conectando os dois serviços onde a equipe cirúrgica a distância que tinha como objetivo repetir o mesmo exame do modo tradicional para confirmação dos resultados. O clínico geral realizava os exames físicos orientados pelo cirurgião que o acompanhava via teleconferência. Este sistema apresentou coincidência de resultados em 48 dos 50 casos. O estudo concluiu que as pacientes sentiramse satisfeitas por evitar o desconforto físico de uma viagem à um distante centro de referência e que houve concordância suficiente para seu uso clínico a distância.

O trabalho realizado no Departament of Acute Critical Medicine Osaka Medical School (Osaka - Japão), descreveu um sistema de telecomunicação para fins de telemedicina, e a avaliação de sua utilidade clínica como suporte diagnóstico em imagens radiológicas e endoscópicas. $\mathrm{O}$ sistema foi testado 53 vezes no Japão e 5 vezes em procedimentos trans-oceânicos. $\mathrm{O}$ artigo concluiu que o sistema mostrou-se eficiente para o apoio diagnóstico feito por especialistas fora do local do atendimento ${ }^{(10)}$.

\section{MÉTODOS}

1 - Equipamentos utilizados: Lâmpada de fenda Topcon ${ }^{\circledR}$ (EUA), modelo SL2ED; divisor de luz com adaptador para câmeras digitais modelo TL51 Topcon ${ }^{\circledR}$ (EUA); câmera digital LG $^{\circledR}$ Honeywell (Coréia) Model N GC 405 N-G Color Camera, $1 \mathrm{CCD}$; placa de captura de imagens Matrox Meteor II ${ }^{\circledR}$ (Matrox
Imaging-EUA); Slot PCI; computador pessoal Pentium ${ }^{\circledR} 550 \mathrm{Mhz}$, trabalhando com o sistema operacional Windows e o software Eyemail;

2 - Modo de operação: Antes de iniciar o exame oftalmológico na lâmpada de fenda, o modo de captura de imagens do programa Eyemail ${ }^{\mathbb{R}}$ deverá ser acionado com um clique no ícone "capturar imagens". O exame de biomicroscopia deverá ser executado com observação da imagem no monitor. As imagens de boa qualidade das estruturas importantes para o caso clínico poderão ser capturadas e congeladas com um clique no mouse, ou por uma pressão no pedal (conectado à entrada do joystick). Durante o exame digital o médico deverá fixar sua atenção no monitor de vídeo para acompanhar as imagens, que após a captura serão arquivadas automaticamente. Caso seja necessário acrescentar informações às imagens, o médico poderá acessar o ícone "detalhes da ficha" (Fig. 1) e digitar as informações necessárias.

3 - Ferramenta digital;

3.1 - Nome do software: Eyemail ${ }^{\circledR}$, Versão Atual: 1.0.0.0;

3.2 - Idealizadores: Alessandro Dantas Pennella, Paulo Schor;

3.3 - Desenvolvedor: ABC Software- Linhares(ES); Programador/analista: Adilson Bragança Cápua Júnior;

3.4 - Plataforma: IBM-PC \& compatíveis, sistemas operacionais: Microsoft Windows 95/98/ME/XP ${ }^{\circledR}$;

3.5 - Linguagem: Imprise/Borland Delphi 5 Client/Server ${ }^{\circledR}$.

3.6 - Banco de Dados: DBISAM 3.02 (Elevate Software);

3.7 - Formato de Armazenamento Interno: Bitmap 24 bit's True color(BMP);

3.8 - Formato de envio para website: Joint Photographic Expert Group(JPEG).

3.9 - Padrão de transferência: FTP(File Transfer Protocol) \& e-mail;

3.10 - Domínio virtual de apoio: www.suportemedico.com.br

3.11 - Período de desenvolvimento:janeiro/2001-novembro/2001.

4 - Funcionamento: O Eyemail ${ }^{\mathbb{R}}$ é um software de captura, armazenamento e transmissão de arquivos de textos e imagens, permitindo que sejam capturadas imagens produzidas

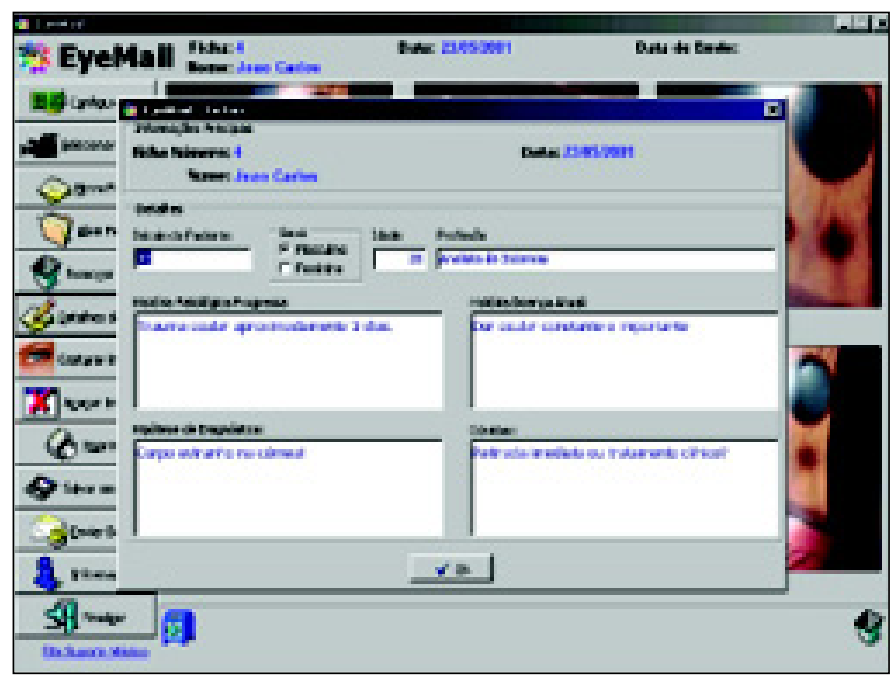

Figura 1 
por qualquer fonte compatível com o sistema Twain (como uma câmera digital, por exemplo), presente em todas as versões do Windows ${ }^{\circledR}$. O Eyemail ${ }^{\circledR}$ permite, através de módulos de gerenciamento, a organização do conjunto de dados (imagens + informações escritas) de diversos exames. Cada conjunto de informações é armazenado em uma ficha de registros que pode estar relacionada com fichas contendo a descrição de uma patologia, história familiar e hipótese diagnóstica (Fig. 1).

Utilizando o mouse é possível capturar imagens e transferilas para a internet. O Eyemail armazena imagens no formato BMP(Bitmap) de 24 bit's. No entanto, antes das imagens serem transferidas para o domínio virtual elas são convertidas, em tempo real, para o formato JPEG. Com a conversão o tamanho das imagens em Bitmap tem um tamanho médio de $750 \mathrm{k}$, tem o tamanho dos seus arquivos reduzidos em quase $70 \% \mathrm{e}$

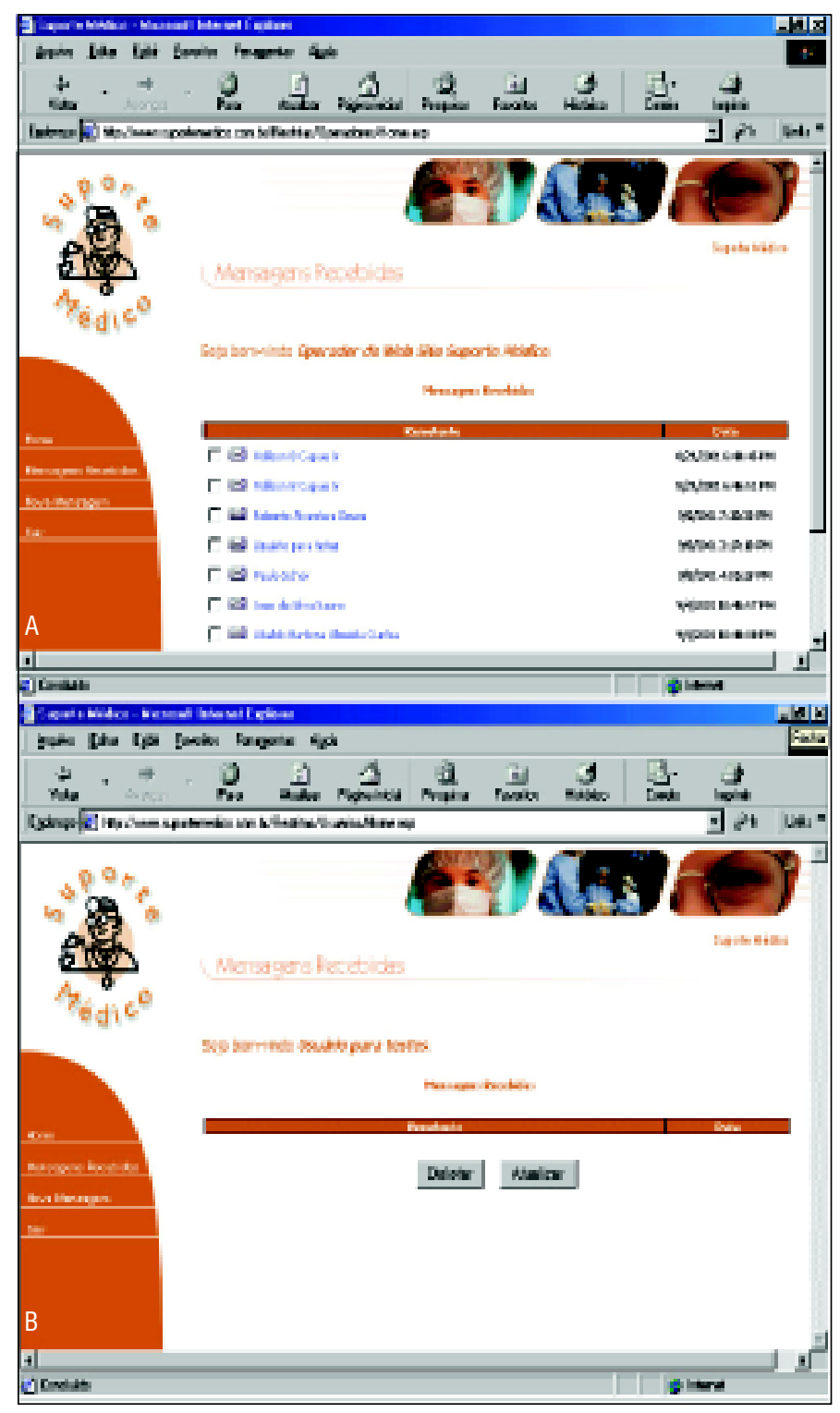

Figura 2 passam para aproximadamente $90 \mathrm{k}$, em JPEG com uma imagem de aproximadamente $90 \mathrm{dpi}$ e $20 \mathrm{~cm}$ de tamanho real e a sua qualidade em aproximadamente $10 \%$. Concomitante ao envio das informações para o domínio virtual, uma mensagem de alerta via e-mail é enviada ao dispositivo móvel do operador de plantão (pager, telefone celular, etc) alertando-o da chegada do pedido de consultoria. O Eyemail ${ }^{\circledR}$ possui uma interface intuitiva permitindo utilização de todos os recursos sem conhecimentos profundos de programação.

5 - Ambiente na internet

5.1 - Domínio virtual: www.suportemedico.com.br

5.2 - Desenvolvimento: Negocios.com - Soluções Web Linhares (ES);

5.3 - WebMaster: Gissele Locatelli;

5.4 - Idealizadores: Alessandro Dantas Pennella, Paulo Schor.

5.5 - Funções: O domínio virtual www.suportemedico.com.br foi desenvolvido de modo a permitir o acesso dos usuários ao sistema de segunda opinião. Divide-se em duas áreas, uma de acesso restrito ao consultor (fig. 2A) a e a outra de acesso restrito ao usuário (fig. 2B).

6 - Recursos do usuário e do consultor: O usuário tem a possibilidade de enviar casos clínicos com imagens e textos diretamente pela internet utilizando imagens previamente digitalizadas que serão anexadas à solicitação de consultoria. $\mathrm{O}$ acesso às mensagens enviadas pelo consultor será feito diretamente pelo domínio virtual, na página do usuário, no ícone "mensagens recebidas". O recurso de discussão do caso clínico com o operador, via interação virtual escrita, poderá ser utilizado acessandose na página principal o ícone "chat". O operador de plantão pode prestar sua consultoria de qualquer computador ligado à internet acessando a sua área no domínio virtual.

6.1 - Provedor de hospedagem: Intercol-Internet Colatina Ltda - Colatina (ES); website - www.intercol.com.br.

6.2 - Desenvolvimento - Plataforma do website: Linguagem ASP $^{\circledR}$ (Active Server Pages); Banco de Dados MS Access ${ }^{\circledR}$.

\section{RESULTADOS}

A funcionalidade do programa Eyemail foi verificada utilizando-se um usuário na cidade de Linhares, Espírito Santo e um operador na cidade de São Paulo, São Paulo. O conjunto de informações com imagens do segmento anterior e informações textuais de seis casos clínicos de pacientes com blefarite, corpo estranho na córnea e conjuntivite, foram enviados com sucesso para o website de consultoria, com o formato HTML. Após o envio foi utilizado um atalho do próprio software para se acessar o website www.suportemedico.com.br. Concomitantemente ao envio das informações, uma mensagem de alerta foi emitida, com sucesso, via e-mail, para o telefone celular do operador do sistema, avisando-o da mensagem. O operador, acessou o website para estudar os casos e responder as dúvidas do usuário. As imagens foram enviadas ao software sem intercorrências. O operador do website considerou que as imagens adicionadas ao texto foram suficientes para realizar os diagnósticos. 


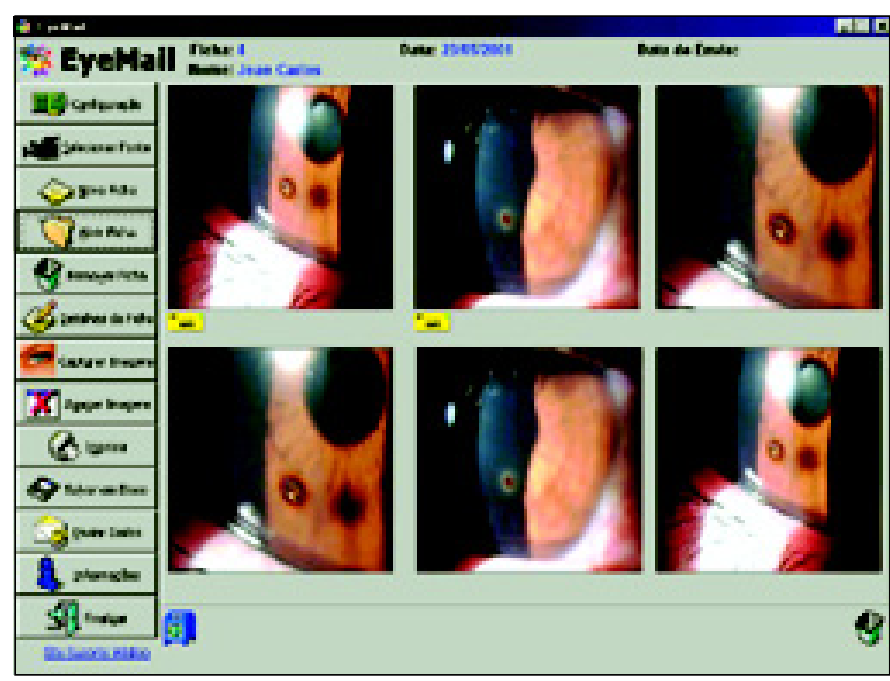

Figura 3

\section{DISCUSSÃO}

A telemedicina é uma área em franco desenvolvimento que irá permitir um suporte diagnóstico para consultorias a distância como no estudo piloto relatado acima. O sistema mostrouse simples e de fácil manuseio, e a obtenção das imagens durante o exame exigiu poucas modificações na técnica habitual de biomicroscopia. A utilização da lâmpada de fenda acoplada à câmera digital mostrou-se adequada para obtenção de imagens das estruturas do segmento anterior e das pálpebras. O tipo de iluminação, sem flash, não é ideal para fotografias, o que nos dificultou na aquisição de imagens de excelente qualidade. Tal fato deverá ser motivo de aprimoramento nas futuras versões do sistema integrado.

Com o desenvolvimento e barateamento da velocidade de conexão à internet (atualmente em torno de $350 \mathrm{kbps}$ com banda larga), passamos a ter a possibilidade de trocar arquivos volumosos em tempo real. Assim, uma teleconsultoria médica poderá ser realizada no futuro em tempo real, como um vídeo chat para a discussão dos casos, como o sistema de videoconferência ${ }^{(10)}$. Estamos, porém, em um pais com limitações importantes de tráfego de informações na rede e restrições de orçamento, motivo pelo qual consideramos adequado um sistema que contempla tais fatos, como o apresentado neste artigo, não optando por sistemas de videoconferência de alto custo ${ }^{(5,9)}$.

As imagens foram enviadas através do software ao website sem perda significativa da sua qualidade, sendo possível a identificação das estruturas oculares normais e das alterações patológicas em todos os seis casos enviados.

O suporte médico é um projeto piloto e com pequena capacidade de armazenamento e transmissão de dados, não sendo possível, no estágio atual, ser utilizado em grande escala. Para tanto teremos de adaptar sistemas criptográficos para evitar o acesso de pessoas não autorizadas às informações veiculadas, além de desenvolver sistemas de controle de aces- so e backup mais sofisticados. A necessidade de um sistema confiável e uma equipe de consultoria de excelente nível técnico dificultaria a implementação deste sistema fora dos serviços universitários de referência, sendo nossa opção desenvolvê-lo na UNIFESP.

\section{CONCLUSÃO}

O sistema foi adequadamente idealizado e desenvolvido, sendo verificada a funcionalidade do mesmo. Sua utilidade potencial ao telediagnóstico e segunda opinião, porém ainda necessita de testes clínicos e comparação com métodos diagnósticos tradicionais para comprovar a sua eficácia.

\section{ABSTRACT}

Purpose: To investigate the possibilities of a teleophthalmology system to be used in a remote second referral program. Methods: Digital images of the anterior segment were digitized by the Eyemail program and attached to the clinical description of the case and the file was sent to the website www.suportemedico.com.br for remote telediagnosis. The telediagnosis was then compared with the in loco diagnosis. Results: The Eyemail system was able to capture images from the slit lamp and to send the files to the website. The website received the files and the operator was able to make the diagnosis using those files. Conclusion: The Eyemail system proved to have a potential use as a second referral program.

Keywords: Telemedicine; Diagnosis, computer-assisted; Computer communication networks

\section{REFERÊNCIAS}

1. Feldman EL: Digital fluorescein tele-angiography. Am J Ophthalmol 1987; 104:91-3.

2. Garden JW, Knapp CF, Saunders JH: Ophtalmologic eletronic imaging and data transfer. J Ky Med Assoc 1991;89:115-7.

3. Bellazzi R, Montani S, Riva A, Stefanelli M. Web-based telemedicine systems for home-care: technical issues and experiences. Comput Methods Programs Biomed 2001;64;3:175-87.

4. de Lusignan S, Althans A, Wells S, Johnson P, Vanderburg M, Robinson J. A pilot study of radiotelemetry for continous cardiopulmonary monitoring of patients at home. J. Telemed Telecare 2000;6suppl(1):S119-22.

5. Barnard CM, Goldyne ME. Evaluation of an asynchronous teleconsultation system for diagnosis of skin cancer and other skin diseases. Telemed J E Health 2000;6:379-84.

6. Burgul R, Gilbert FJ, Undrill PE. Methods of measurement of image quality in teleultrasound. Br. J. Radiol 2000;73:1306-12.

7. Li HK. Telemedicine and ophthalmology. Surv Ophtalmol 1999;44:61-72.

8. Wada M, Suzuki R, Murakami T. Super-high-definition image systems for telemedicine. J Telemed Telecare 2000;6Suppl(2):S85.

9. Aarnio P, Jaatinen P, Hakkari K, Halin N. A new method for surgical consultations with videoconference. Ann Chirur Gynaecol 2000;89:336-40.

10. Ueda T, Hida S, Tanaka H, Kumagai Y, Kudou T, Shimazu T, Sugimoto H Telemedical support using real-time ultrasonography and endoscopy images. Comput Methods Programs Biomed 2001;66:55-61. 